\title{
HAADF/MAADF Observations and Image Simulations of Dislocation Core Structures in a High Entropy Alloy
}

\author{
T.M. Smith ${ }^{1}$, B.D. Esser ${ }^{1}$, E.P. George ${ }^{2}$, F. Otto ${ }^{2}$, M. Ghazisaeidi ${ }^{1}$, D.W. McComb ${ }^{1}$, M.J. Mills ${ }^{1}$ \\ 1. Center for Electron Microscopy and Analysis, The Ohio State University, Columbus, OH \\ 2. Institute for Materials, Ruhr University, Bochum, Germany
}

High entropy alloys (HEAs) are a new class of multi-component alloys in which the individual elements have similar concentrations. A single-phase solid solution HEA containing 5 elements $(\mathrm{Co}, \mathrm{Cr}, \mathrm{Fe}, \mathrm{Mn}$, and $\mathrm{Ni}$ ) with equiatomic composition was first discovered by Cantor [1]. Among the surprising characteristics of this fcc HEA are: strong temperature dependence of the yield strength at temperatures around and below room temperature, relatively weak strain-rate dependence over the same temperature range [3]; very large hardening rates [2,3]; and large fracture toughness at room temperature [4]. These features are linked to deformation twinning and dislocation-mediated plasticity, yet presently there is insufficient knowledge of dislocation dissociation, stacking fault energy, or core structures in this alloy. The highly planar deformation involves dislocation arrays on active slip systems (Figure 1a and 1b). This characteristic could imply the presence of short range order, low fault energy, or supplementary displacements in the wake of glide dislocations.

In the present study, an HEA sample, with the same nominal composition as above, was deformed to 5\% plastic strain at room temperature. Post-mortem electro-polished $3 \mathrm{~mm}$ disks were extracted and analyzed using a probe-corrected Titan $\mathrm{G}^{3} 80-300 \mathrm{kV}$ along a [110] Zone axis. Figure 2a shows a high angle annular dark field (HAADF) image of a $1 / 2<110>60^{\circ}$ mixed dislocation obtained at a short camera length $(93 \mathrm{~mm})$. A notable observation is a $\sim 3 \mathrm{~nm}$ wide stacking fault, suggesting a modest stacking fault energy (SFE) in the alloy. Subsequent observations revealed a range in stacking fault lengths (2$8 \mathrm{~nm}$ ), possibly due to local chemical fluctuations affecting the SFE throughout the solid solution. Conversely, the edge dislocation shown in Figure 2c is a $1 / 2[1-10]$ Lomer dislocation, exhibiting an extremely compact structure, with no noticeable dissociation into a Lomer-Cottrell configuration. This dislocation is inconsistent with the $60^{\circ}$ dislocation with respect to apparent stacking fault energy. The medium angle annular dark field (MAADF) images in Figure $\mathbf{2 b}$ and $\mathbf{2 d}$, taken at longer camera lengths (145-230mm), contain stronger diffraction effects [5]. Zones of enhanced intensity ("plumes") can be observed in the MAADF, but not the HAADF images, implying the intensity is not a consequence of elemental segregation around the dislocation cores. Significant variations in the shape, size, and location of the "plumes" were observed around a dislocation core. Understanding the cause of these "plumes" can give insights into the structure of the dislocation cores through the thickness of the sample, and stimulate new ideas about how dislocations influence the surrounding lattice as they glide.

The fact that the "plumes" are not observed in HAADF but appear and become brighter at increasingly longer camera lengths reveals that the contrast is either a natural consequence of: (a) the deviation from a perfect lattice near the dislocation core, (b) a slight misalignment either due to overall rotation of the dislocation in the thin foil or variation in the local separation distance between partials, or (c) a variation in the dissociation through the depth in the sample [6]. Atomistic simulations on simple systems were used to test the above possibilities systematically. Cells containing the dislocation cores corresponding to (a)-(c) were created. HAADF and MAADF images of these cells were then simulated using the $\mu \mathrm{STEM}$ multislice code [7] along with zone axis STEM simulations using CTEM soft[8]. The findings 
from these simulations combined with through-focus acquisitions to observe imaging effects through thickness will be discussed in detail.

References:

[1] B. Cantor et al. Mat Sci Eng a-Struct, 375 (2004) 213-218.

[2] F. Otto et al. Acta Materialia, 61 (2013) 5743-5755

[3] A. Gali, E.P. George. Intermetallics, 39 (2013) 74-78.

[4] B. Gludovatz et al. Science, 345 (2014) 1153-1158.

[5] P.J. Phillips et al. Ultramicroscopy, 116 (2012) 47-55.

[6] J.G. Lozano et al. Physical Review Letters, 113 (2014). 135103

[7] B.D. Forbes et al. Physical Review B, 82 (2010) 104103.

[8] P.J. Phillips, M.J. Mills, M. De Graef. Philosophical Magazine, 91 (2011) 2081-2101.

[9] Support for this work was provided by the US Department of Energy, Office of Basic Energy Sciences under Grant \#DE-SC0001258 and the Center for Emergent Materials: an NSF MRSEC under award number DMR-1420451. This work was also supported in part by an allocation of computing time from the Ohio Supercomputer Center.

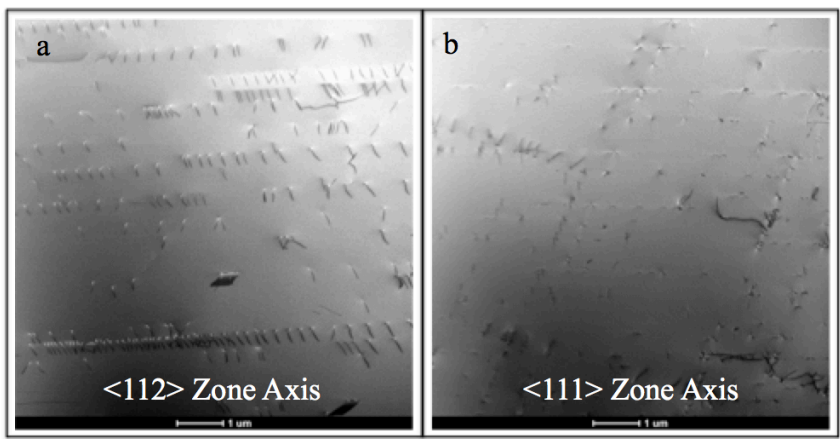

Figure 1: (a) $<112>$ Zone Axis BF (S)TEM image of dislocation arrays in a HEA (b) $<111>$ Zone Axis $\mathrm{BF}(\mathrm{S}) \mathrm{TEM}$ image of dislocation arrays in a HEA.
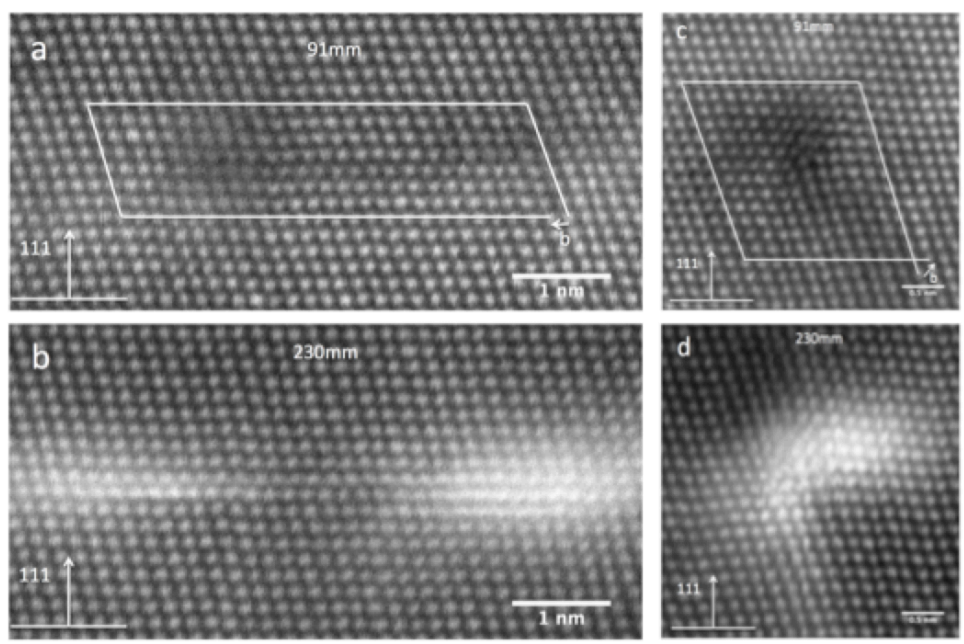

Figure 2: STEM images of dislocations in the HEA at two different camera lengths (HAADF vs. MAADF conditions). (a) and (b) are the same $1 / 2<110>\{111\} 60^{\circ}$ dislocation; (c) and (d) are the same $1 / 2<110>\{001\}$ Lomer dislocation. Origin of the "plumes" in the MAADF images is presently unknown. 